\title{
Discrete Dynamics of Complex Systems
}

\author{
HERMANN HAKEN \\ Institute for Theoretical Physics and Synergetics, University of Stuttgart, Pfaffenwaldring 57/4, D-70550 Stuttgart, Germany
}

(Received 9 October 1996)

\begin{abstract}
This article extends the slaving principle of synergetics to processes with discrete time steps. Starting point is a set of nonlinear difference equations which contain multiplicative noise and which refer to multidimensional state vectors. The system depends on a control parameter. When its value is changed beyond a critical value, an instability of the solution occurs. The stability analysis allows us to divide the system into stable and unstable modes. The original equations can be transformed to a set of difference equations for the unstable and stable modes. The extension of the slaving principle to the time-discrete case then states that all the stable modes can be explicitly expressed by the unstable modes or so-called order-parameters.
\end{abstract}

Keywords: Discrete dynamics, Difference equations, Noise, Synergetics, Slaving principle, Complex systems

\section{INTRODUCTION}

When we look at the various scientific disciplines, quite generally speaking we can observe the following main trends:

1. Instead of describing and studying states of a system, science is more and more interested in their temporal evolution, or, in other words, in their dynamics.

2. There is a tendency of an ever increasing mathematization of disciplines.

3. So-called linear laws must be replaced by nonlinear laws. In most cases the dynamics of systems has been studied in the frame of evolution equations that in one way or another were modeled in analogy to basic laws of physics, which describe the temporal evolu- tion of the state of a system, for instance of its electro-magnetic fields. In physics, space, time and (classical) variables are usually treated as continuous quantities.

When we treat realistic cases in population dynamics, economy, ecology, biology, and many other fields, the continuous approach to space and time and to the state variables becomes, however, questionable, at least in a number of cases. But even in physics it can become desirable (or necessary) to look for discrete approaches. In classical physics we are confronted with discrete maps when we think of the Poincare crosssection. Let us assume that we can define the trajectories in a multidimensional space whereby the trajectories develop in the course of continuous time $t$. In order to reduce the dimensionality 
of the system, one may introduce a hyperplane. Then the cross-sections of the trajectories with that hyperplane can be denoted by $\mathbf{x}_{l}$ and a relationship between subsequent cross-sections $\mathbf{x}_{l+1}$ and $\mathbf{x}_{l}$ be established. In this way, a continuous transformation is replaced by a discrete map. Instead of deriving the law that connects $\mathbf{x}_{l+1}$ with $\mathbf{x}_{l}$ from the original dynamics, one may establish such laws also in the form of models. An example, where continuous state variables must be replaced by discrete ones, is provided by quantum mechanics. For instance, the continuous states of planetary orbits have to be replaced by discrete quantum states in atoms that are labeled by the quantum numbers $n, l, m$. Studying this case further leads to the question in how far processes can be found in quantum systems that carry signatures of chaos. Quantum states play a fundamental role also in quantum chemistry. In addition here we are dealing with discrete numbers of molecules. Modern techniques in chemistry and biochemistry are concerned with the detection of single molecules by appropriate statistical methods of observation. I just refer to Rigler's and Eigen's work. Biology abounds with processes that occur in discrete time or space, or are connected with discrete variables. Just think of the discrete firing of neurons in the brain, sensory processes, for instance absorption of individual photons in the retina, the formation of discrete cells, or discrete bone sections, such as in the skeleton. Regional planning abounds with discrete processes in space and time, for instance parcellation of land, and so on.

Quite evidently, this list is inexhaustible and may reach from rather trivial cases to very sophisticated problems in which discreteness plays a fundamental role. Discreteness can give rise to instabilities that are otherwise not present, as is, for instance, well known from the numerical solution of partial differential equations. Thus, there is certainly a big demand for the development of concepts and mathematical methods that deal with discrete systems. The discreteness may be in space, where space is not distributed con- tinuously but divided into individual cells. A typical example of this approach is the cellular automaton. The dynamics need not proceed in continuous time, for instance observations may be done at discrete time, or the discreteness of time is inherent in the system. Finally the variables describing a system may be discrete.

While in earlier times it has been quite often believed that the transition from a discrete description in either of these cases to a continuous description does not lead to qualitative differences, it is now well established that quite decisive differences in the behavior of a system may occur. The probably most famous example of this kind is the logistic equation, whose solutions show a very rich chaotic behavior depending on a single parameter value [1], whereas the corresponding continuous case shows just one kind of simple solution. There is still another more formal reason for studying discrete systems, namely the use of digital computers. Here both time and the state variables must be described as discrete variables. The question arises whether we can reformulate hitherto known concepts and mathematical approaches so to cope with discrete variables or whether entirely new methods will be needed. This is quite obviously a vast field to be explored. In my paper I want to address the question of how the slaving principle of synergetics [2] can be transferred from the continuous to the discrete time case.

In view of the tremendous number of different systems including model systems in the different scientific disciplines and the great variety of phenomena they show, it might seem to be an idle question whether there are general basic laws or features underlying the behavior of such systems. $\mathrm{We}$ are here mainly thinking of the so-called complex systems that are composed of many individual parts. Over the past decades, it has become more and more transparent that in spite of the diversity of such systems a number of general principles and laws can be found provided we focus our attention on situations in which the system's behavior changes qualitatively on a 
macroscopic scale. As was shown in the continuous time case [2], in situations, where the macroscopic qualitative behavior of a system changes, the dynamics of the system is governed by few variables, the so-called order parameters. Once these variables are identified, one may deduce the behavior of the individual parts of a system via the slaving principle, which has a number of special cases, such as the center manifold theorem. The resulting order parameter equations can be grouped into classes each of which shows typical behavior. An example for such a class is the Ginzburg-Landau equation, other classes can be identified by suitable generalizations of these equations. Another example is provided by the nonlinear Schrödinger equation. It is by now well known that quite different systems in a variety of disciplines obey again and again the same kind of nonlinear equations. In this way one can draw conclusions from one field to another one. As was pointed out by Mikhailov [3], synergetics can be considered as a bag of model equations that can be applied to a variety of fields. Thus the strength of the synergetic approach consists in drawing analogies between quite different complex systems so that we get more and more acquainted with the behavior of such systems irrespective of their material nature. The question arises, of course, whether the research strategy of synergetics can be transferred to the case of discrete dynamics. Equations that describe the evolution of a system with discrete time steps are quite often also called maps. The study of such maps has become a modern most lively branch of mathematics but also of other disciplines.

In the following I wish to give an explicit example of how the slaving principle can be transferred to discrete noisy maps, i.e. the discrete time evolution. Thus this example may indicate how research on discrete processes may occur in the future. But to be sure, here we are standing at the beginning of an enormously wide field of research which will provide us with many surprising results.

\section{DISCRETE NOISY MAPS}

Let us consider a dynamical system described by a state vector $\mathbf{q}_{l}$, which is defined at a discrete and equidistant time sequence $l$. Actually the requirement of equidistancy is not necessary; we just have to relabel the time sequence $l$ by $t_{l}$. The evolution of the system from one discrete time $l$ to the next $l+1$ is described by an equation of the geneal form

$$
\mathbf{q}_{l+1}=\mathbf{f}\left(\mathbf{q}_{l}, l\right)+G\left(\mathbf{q}_{l}, l\right) \eta_{l} .
$$

Here $\mathbf{f}$ and $G$ are nonlinear functions of $\mathbf{q}_{l}$ and may depend on the index $l$ explicitly. The first part on the r.h.s. of (1) describes a deterministic process, while the second part of the right-hand side describes random events. In the second part $\eta_{l}$ is a random vector, whose probability distribution may-but need not-depend on the index $l$. The first steps of our analysis are entirely analogous to those in the case where time proceeds continuously. We assume that for a control parameter $\alpha_{0}$ a solution - that is time-independent - is known

$$
\alpha_{0}: \mathbf{q}^{0} \text {. }
$$

Without loss of generality, we may assume

$$
\mathbf{q}^{0}=0 \text {. }
$$

In order to check the stability of the solution (2), (3), when the control parameter value is changed to a new one, $\alpha$, we keep only the linear terms of Eq. (1) and neglect the fluctuating part

$$
\mathbf{q}_{l+1}=L \mathbf{q}_{l} .
$$

Making the hypothesis

$$
\mathbf{q}_{l}=V \xi_{l},
$$

where $V$ is a matrix, we can cast (4) into the form

$$
\xi_{l+1}=\hat{L} \xi_{l}
$$

with

$$
\hat{L}=V^{-1} L V \text {. }
$$


We can choose $V$ in such a way that $\hat{L}$ is brought into Jordan's normal form. Depending on the size of the eigenvalues of $\hat{L}$,

$$
\begin{aligned}
& |\lambda| \geq 1, \\
& |\lambda|<1,
\end{aligned}
$$

we may distinguish between unstable modes $\mathbf{u}$ and stable modes $\mathbf{s}$, respectively. In terms of these new mode amplitudes, the original equations (1) can be cast into the form

$$
\mathbf{u}_{l+1}-\mathbf{u}_{l}=\Lambda_{u} \mathbf{u}_{l} \mathrm{~d} l+\mathrm{d} \mathbf{Q}(\mathbf{u}, \mathbf{s}, l)
$$

and

$$
\mathbf{s}_{l+1}-\mathbf{s}_{l}=\Lambda_{s} \mathbf{s}_{l} \mathrm{~d} l+\mathrm{d} \mathbf{P}(\mathbf{u}, \mathbf{s}, l)
$$

We assume $\Lambda_{s}$ in Jordan's normal form with negative diagonal elements. For simplicity, we also assume that $\Lambda_{u}$ is diagonal and of smallness $\delta$, but the procedure can easily be extended to a more general $\Lambda_{u}$.

Note that $\mathrm{d} \mathbf{Q}, \mathrm{d} \mathbf{P}$ may contain $\mathbf{u}$ and $\mathbf{s}$ also at retarded times $\mathbf{u}$, so that they are functions of $\mathbf{u}_{l}, \mathbf{u}_{l-1} \ldots$ From our result it will transpire that we can also allow for a dependence of $d \mathbf{Q}$ and $\mathrm{d} \mathbf{P}$ on future times, i.e., for instance, on $\mathbf{u}_{l+1}$. Our purpose is to devise a procedure by which we can express $\mathbf{s}$ in a unique and well-defined fashion by $\mathbf{u}$ and $l$ alone. Let us therefore assume that such a replacement can be made. This allows us to establish a number of formal relations used later on.

\section{SOME FORMAL RELATIONS}

In a first step of our analysis we shall assume that $\mathbf{s}$ can be expressed by a function of $\mathbf{u}$ and $l$ alone. This allows us to consider $\mathrm{d} \mathbf{P}$ in (11) as a function of $\mathbf{u}$ and $l$ alone

$$
\mathbf{s}_{l+1}-\mathbf{s}_{l}=\Lambda_{s} \mathbf{s}_{l} \mathrm{~d} l+\mathrm{d} \mathbf{P}\left(\mathbf{u}_{l}, \mathbf{u}_{l-1}, \ldots, l\right) .
$$

This equation can be solved by

$$
\mathbf{s}_{l+1}=\sum_{m=-\infty}^{l}\left(1+\Lambda_{s} \mathrm{~d} m\right)^{l-m} \mathrm{~d} \mathbf{P}\left(\mathbf{u}_{m}, \mathbf{u}_{m-1}, \ldots, m\right),
$$

as can be checked by inserting (13) into (12). As transpires from (13), $\mathbf{s}_{l+1}$ is represented by a sum that contains $\mathbf{u}_{m}, \ldots$ at all former times. On the other hand, in the case of continuous time, the slaving principle allows one to express $\mathbf{s}$ as a function of $\mathbf{u}$ at the same time. Therefore we seek a formalism that allows us to express $\mathbf{s}_{l+1}$ by as little past time steps of $\mathbf{u}$ as possible. In order to achieve this goal we first establish several formal relations. We introduce the abbreviation

$$
\Delta_{-} \mathbf{s}_{l+1}=\mathbf{s}_{l+1}-\mathbf{s}_{l}
$$

by which we can express $\mathbf{s}_{l}$ by means of $\mathbf{s}_{l+1}$

$$
\mathbf{s}_{l}=\left(1-\Delta_{-}\right) \mathbf{s}_{l+1} .
$$

This allows us to write (12) in the form

$$
\left(\Delta_{-}-\Lambda_{s} \mathrm{~d} l\left(1-\Delta_{-}\right)\right) \mathrm{s}_{l+1}=\mathrm{dP}\left(\mathrm{u}_{l}, l\right) .
$$

The reader is reminded that $\mathrm{d} \mathbf{P}$ may be a function of $\mathbf{u}$ at various time indices $l, l-1, \ldots$

$$
\mathrm{d} \mathbf{P}\left(\mathbf{u}_{l}, l\right)=\mathrm{d} \mathbf{P}\left(\mathbf{u}_{l}, \mathbf{u}_{l-1}, \ldots, l\right) .
$$

The formal solution of (16) reads

$$
\mathbf{s}_{l+1}=\left(\Delta_{-}\left(1+\Lambda_{s} \mathrm{~d} l\right)-\Lambda_{s} \mathrm{~d} l\right)^{-1} \mathrm{~d} \mathbf{P}\left(\mathbf{u}_{l}, l\right) .
$$

A comparison of this solution with the former solution (13) yields

$$
\begin{array}{r}
\left(\Delta_{-}\left(1+\Lambda_{s} \mathrm{~d} l\right)-\Lambda_{s} \mathrm{~d} l\right)^{-1} \mathrm{~d} \mathbf{P}\left(\mathbf{u}_{l}, l\right) \\
\quad=\sum_{m=-\infty}^{l}\left(1+\Lambda_{s} \mathrm{~d} m\right)^{l-m} \mathrm{~d} \mathbf{P}\left(\mathbf{u}_{m}, m\right) .
\end{array}
$$

In the following we wish to evaluate the 1.h.s. of (19) in a way that in the continuous time case 
would correspond to a partial integration and thus allows us to reduce the recurrency operation. We now introduce the decomposition

$$
\Delta_{-}=\Delta_{-}^{(l)}+\Delta_{-}^{(u)} T_{-}^{(l)},
$$

where $\Delta_{-}^{(l)}, \Delta_{-}^{(u)}, T_{-}^{(l)}$ operate as follows:

$$
\begin{gathered}
\Delta_{-}^{(l)} f\left(\mathbf{u}_{l}, l\right)=f\left(\mathbf{u}_{l}, l\right)-f\left(\mathbf{u}_{l}, l-1\right), \\
\Delta_{-}^{(u)} f\left(\mathbf{u}_{l}, l\right)=f\left(\mathbf{u}_{l}, l\right)-f\left(\mathbf{u}_{l-1}, l\right), \\
T_{-}^{(l)} f\left(\mathbf{u}_{l}, l\right)=f\left(\mathbf{u}_{l}, l-1\right) .
\end{gathered}
$$

Using some little algebra, one readily establishes the following identity:

$$
\begin{aligned}
\left\{\Delta_{-}\right. & \left.\left(1+\Lambda_{s} \mathrm{~d} l\right)-\Lambda_{s} \mathrm{~d} l\right\}^{-1} \\
= & \left\{\Delta_{-}^{(l)}\left(1+\Lambda_{s} \mathrm{~d} l\right)-\Lambda_{s} \mathrm{~d} l\right\}^{-1} \\
& -\left\{\Delta_{-}\left(1+\Lambda_{s} \mathrm{~d} l\right)-\Lambda_{s} \mathrm{~d} l\right\}^{-1} \cdot[\ldots]
\end{aligned}
$$

where the square bracket represents an abbreviation defined by

$$
\begin{aligned}
{[\ldots]=} & \left(1+\Lambda_{s} \mathrm{~d} l\right) \times \Delta_{-}^{(u)} T_{-}^{(l)} \\
& \times\left\{\Delta_{-}^{(l)}\left(1+\Lambda_{s} \mathrm{~d} l\right)-\Lambda_{s} \mathrm{~d} l\right\}^{-1} .
\end{aligned}
$$

Because of (19), we may expect that the curly brackets in (25) possess an analog to the explicit form of r.h.s. of (19). This is indeed the case.

Let us assume for the moment that we may decompose $d \mathbf{P}$ into a sum of expressions of the form

$$
h\left(\mathbf{u}_{m}, \mathbf{u}_{m-1}, \ldots\right) \mathrm{d} \mathbf{g}(m),
$$

where $h$ is a function of the variables $\mathbf{u}$ alone, but does not depend explicitly on $m$, whereas dg is a vector which depends on $m$ alone. It is then simple to verify the following relation:

$$
\begin{aligned}
& \sum_{m=-\infty}^{l}\left(1+\Lambda_{s} \mathrm{~d} m\right)^{l-m} h\left(\mathbf{u}_{m}, \mathbf{u}_{m-1}, \ldots\right) \mathrm{d} \mathbf{g}(m) \\
& \quad=h\left(\mathbf{u}_{l}, \mathbf{u}_{l-1}, \ldots\right) \sum_{m=-\infty}^{l}\left(1+\Lambda_{s} \mathrm{~d} m\right)^{l-m} \mathrm{~d} \mathbf{g}(m)
\end{aligned}
$$

$$
\begin{aligned}
& -\sum_{m=-\infty}^{l}\left(1+\Lambda_{s} \mathrm{~d} m\right)^{l+1-m} \\
& \times \sum_{m^{\prime}=-\infty}^{m-1}\left(1+\Lambda_{s} \mathrm{~d} m\right)^{m-1-m^{\prime}}\{\ldots\}_{m, m^{\prime}},
\end{aligned}
$$

where the brace is defined by

$$
\{\ldots\}_{m, m^{\prime}}=\Delta_{-} h\left(\mathbf{u}_{m}, \mathbf{u}_{m-1}, \ldots\right) \mathrm{d} \mathbf{g}\left(m^{\prime}\right) .
$$

The essence of (27) can be expressed as follows. While 1.h.s. contains $\mathbf{u}$ at all previous times, the first term on r.h.s. contains $\mathbf{u}$ only at those earlier times which are initially present in $h\left(\mathbf{u}_{l}, \mathbf{u}_{l-1}, \ldots\right)$. Thus, instead of an infinite regression we have only a finite regression. From a comparison of (24) with (27) we may derive the following definition:

$$
\begin{gathered}
{\left[\Delta_{-}^{(l)}\left(1+\Lambda_{s} \mathrm{~d} l\right)-\Lambda_{s} \mathrm{~d} l\right]^{-1} \mathrm{~d} \mathbf{P}\left(\mathbf{u}_{l}, \mathbf{u}_{l-1}, \ldots, l\right)} \\
=\sum_{m=-\infty}^{l}\left(1+\Lambda_{s} \mathrm{~d} m\right)^{l-m} \mathrm{~d} \mathbf{P}\left(\mathbf{u}_{l}, \mathbf{u}_{l-1}, \ldots, m\right),
\end{gathered}
$$

where on r.h.s., $\mathbf{u}_{l}, \mathbf{u}_{l-1}$, etc., are kept fixed and the summation runs only over $m$. Eq. (24) with (25) may be iterated, giving

$$
\begin{aligned}
& \left\{\Delta_{-}\left(1+\Lambda_{s} \mathrm{~d} l\right)-\Lambda_{s} \mathrm{~d} l\right\}^{-1} \\
& \quad=\left\{\Delta_{-}^{(l)}\left(1+\Lambda_{s} \mathrm{~d} l\right)-\Lambda_{s} \mathrm{~d} l\right\}^{-1} \cdot(\ldots),
\end{aligned}
$$

where the parentheses on r.h.s. are given by

$$
\ldots=\sum_{\nu=0}^{\infty}[\ldots]^{\nu}
$$

and these square brackets are defined in (25).

As already noted, in practical cases one does not extend the summation to infinity because it is known from a number of applications that few terms suffice. Furthermore, the convergence of the whole series (31) need not hold so that we are dealing here with semiconvergent series. Therefore it is important to give an estimate for the size of the rest term, provided we take only a finite sum. The rest term for r.h.s. of (30) can be 
derived from the formula

$$
\begin{aligned}
\{\ldots\}^{-1} \sum_{\nu=n+1}^{\infty}[\ldots]^{\nu} & =\{\ldots\}^{-1} \sum_{\nu=0}^{\infty}[\ldots]^{\nu}[\ldots]^{n+1} \\
& =\{\ldots\}^{-1}(1-[\ldots])^{-1}[\ldots]^{n+1}
\end{aligned}
$$

While the operator containing $\Delta_{-}^{(l)}$ is defined by (29), we have still to explain in more detail how to evaluate the operator $\Delta_{-}^{(u)}$. It has the following properties:

(1) It acts only on $\mathbf{u}_{l}, \mathbf{u}_{l-1}, \ldots$

(2) For any function $f$ of $\mathbf{u}_{l}$ it is defined by (21)

$$
\Delta_{-}^{(u)} f\left(\mathbf{u}_{l}\right)=f\left(\mathbf{u}_{l}\right)-f\left(\mathbf{u}_{l-1}\right)
$$

If $f$ contains variables at several "times", we may write

$$
\Delta_{-}^{(u)} f\left(\left\{\mathbf{u}_{l}\right\}\right)=f\left(\left\{\mathbf{u}_{l}\right\}\right)-f\left(\left\{\mathbf{u}_{l-1}\right\}\right),
$$

where

$$
\left\{\mathbf{u}_{l}\right\}=\left(\mathbf{u}_{l}, \mathbf{u}_{l-1}, \ldots\right)
$$

denotes the set of variables containing different indices $l$.

(3) For the product of the functions $\nu$ and $\omega$ we readily obtain

$$
\begin{aligned}
& \left.\Delta_{-}^{(u)}\left(\nu\left(\left\{\mathbf{u}_{l}\right\}\right) w\left(\left\{\mathbf{u}_{l}\right)\right\}\right)\right) \\
& =w\left(\left\{\mathbf{u}_{l}\right\}\right) \Delta_{-}^{(u)} \nu\left(\left\{\mathbf{u}_{l}\right\}\right)+\nu\left(\left\{\mathbf{u}_{l-1}\right\}\right) \Delta_{-}^{(u)} w\left(\left\{\mathbf{u}_{l}\right\}\right) .
\end{aligned}
$$

In the following we shall assume that the functions $f$ can be considered as polynomials.

(4) In order to treat (34) still more explicitly, we use (35) and the following rules:

$$
\Delta_{-}^{(u)} u_{l}^{n}=u_{l}^{n}-u_{l-1}^{n}=\left(\sum_{\nu_{1}+\nu_{2}=n-1} u_{l}^{\nu_{1}} u_{l-1}^{\nu_{2}}\right) \Delta_{-} u_{l} .
$$

The r.h.s. may be written in the form

$$
\left(\phi \frac{\partial u_{l}^{n}}{\partial u_{l}}\right) \cdot \Delta_{-} u_{l},
$$

where the factor and parentheses can be considered as a symmetrized derivative, which is identical with the parentheses in (37).

(5) From relations (37), (38), we readily derive for an arbitrary function of $f(u)$ alone

$$
\Delta_{-}^{(u)} f\left(u_{l}\right)=\phi \frac{\partial f\left(u_{l}\right)}{\partial u_{l}} \cdot \Delta_{-}^{(u)} u_{l},
$$

or, more generally,

$$
\Delta_{-}^{(u)} \mathbf{f}\left(\mathbf{u}_{l}, \ldots, \mathbf{u}_{l^{\prime}}\right)=\sum_{\nu=0}^{l=l^{\prime}} g_{\nu}\left(\mathbf{u}_{l}, \ldots, \mathbf{u}_{l^{\prime}-1}\right) \Delta_{-} \mathbf{u}_{l^{\prime}+\nu}
$$

We may now replace $\Delta_{-} \mathbf{u}_{l^{\prime}+\nu}$ according to (10) by

$$
\Delta_{-}^{(u)} \mathbf{u}_{l^{\prime}+\nu}=\Lambda_{u} \mathrm{~d} l \mathbf{u}_{l^{\prime}+\nu}+\mathrm{d} \mathbf{q}\left(\ldots, l^{\prime}+\nu-1\right),
$$

where we abbreviate the r.h.s. by

$$
\mathrm{d} \overline{\mathbf{Q}}\left(\ldots, l^{\prime}+\nu\right)
$$

This gives (40) in its final form

$$
\begin{aligned}
& \Delta_{-}^{(u)} \mathbf{f}\left(\mathbf{u}_{l}, \ldots, \mathbf{u}_{l^{\prime}}\right) \\
& \quad=\sum_{\nu=0}^{l=l^{\prime}} g_{\nu}\left(\mathbf{u}_{l}, \ldots, \mathbf{u}_{l^{\prime}-1}\right) \mathrm{d} \overline{\mathbf{Q}}\left(\ldots, l^{\prime}+\nu\right) .
\end{aligned}
$$

For sake of completeness, we derive a formula for

$$
\Delta_{-}^{(u)} \sum_{\nu_{0}+\cdots+\nu_{x}=n} u_{m+x+1}^{\nu_{x}} \cdots u_{m+1}^{\nu_{0}},
$$

which, according to the definition (33), can be written as

$$
\sum_{\nu_{0}+\cdots+\nu_{x}=n}\left(u_{m+x+1}^{\nu_{x}} \cdots u_{m+1}^{\nu_{0}}-u_{m+x}^{\nu_{x}} \cdots u_{m}^{\nu_{0}}\right) .
$$


It can be rearranged to

$$
\sum u_{m+x}^{\nu_{x}} u_{m+x-1}^{\nu_{x-1}} \cdots u_{m}^{\nu_{1}}\left(u_{m+x+1}^{\nu_{0}}-u_{m}^{\nu_{0}}\right)
$$

where the parentheses can be written in the form

$$
\sum u_{m+x+1}^{\nu_{0}-1-\lambda} u_{m}^{\lambda}\left(u_{m+x+1}-u_{m}\right) .
$$

We therefore find for (44)

$$
\sum_{\nu_{0}+\cdots+\nu_{x+1}=n-1} u_{m+x+1}^{\nu_{x+1}} \cdots u_{m}^{\nu_{0}}\left(u_{m+x+1}-u_{m}\right)
$$

where we may write

$$
\begin{aligned}
\left(u_{m+x+1}-u_{m}\right)= & u_{m+x+1}-u_{m+x}+u_{m+x} \\
& -\cdots+u_{m+1}-u_{m} \\
= & \sum_{\nu=0}^{x} \Delta_{-} u_{m+1+\nu} .
\end{aligned}
$$

The sum in (48) in front of the parentheses can again be considered as the symmetrized derivative, while the parentheses in (48) can be expressed by individual differences $u_{k+1}-u_{k}$.

\section{THE ITERATION PROCEDURE FOR THE DISCRETE CASE}

Taking all the above formulas together we obtain again a well-defined procedure for calculating $\mathbf{s}$ as a function of $\mathbf{u}$ and $l$, provided $\mathrm{d} \mathbf{P}$ is prescribed as a function of $\mathbf{u}$ and $l$ alone. In practice, however, dP depends on $\mathbf{s}$. Therefore we must devise a procedure by which we may express $\mathbf{s}$ by $\mathbf{u}$ and $l$ stepwise by an inductive process.

To this end we introduce a smallness parameter $\delta$. In general $\mathrm{d} \mathbf{Q}$ and $\mathrm{d} \mathbf{P}$ contain a nonstochastic and a stochastic part according to

$$
\begin{aligned}
\mathrm{d} \mathbf{Q} & =\mathbf{Q}_{0}(\mathbf{u}, \mathbf{s}, l) \mathrm{d} l+\mathrm{d} \mathbf{F}_{u}(\mathbf{u}, \mathbf{s}, l), \\
\mathrm{d} \mathbf{P} & =\mathbf{P}_{0}(\mathbf{u}, \mathbf{s}, l) \mathrm{d} l+\mathrm{d}_{s}(\mathbf{u}, \mathbf{s}, l),
\end{aligned}
$$

where for the stochastic part the following decomposition is assumed: $\mathrm{d} \mathbf{F}_{i}(\mathbf{u}, \mathbf{s}, l)=M_{i}\left(\mathbf{u}_{l}, \mathbf{u}_{l-1}, \ldots, \mathbf{s}_{l}, \mathbf{s}_{l-1}, \ldots\right) \mathrm{d} \mathbf{F}_{0}(l)$,

$$
i=u, s \text {. }
$$

We assume that $\Lambda_{u}$ in (10) is of the order $\delta$ and that the functions occurring in (51), can be expressed as polynomials of $\mathbf{u}$ and $\mathbf{s}$ with $l$-dependent coefficients. The coefficients are either continuous functions (contained in $\mathbf{Q}_{0}$ and $\mathbf{P}_{0}$ ) or quantities describing a Wiener process [in $\left.\mathrm{dF}_{0}(l)\right]$. We assume that the constant terms which are independent of $\mathbf{u}$ and $\mathbf{s}$ are of smallness $\delta^{2}$, the coefficients of the linear terms of smallness $\delta$, while $\mathbf{u}$ and $\mathbf{s}$ are of order $\delta, \delta^{2}$, respectively. In order to devise an iteration procedure, we represent $\mathbf{s}$ in the form

$$
\mathbf{s}(\mathbf{u}, l)=\sum_{k=2}^{\infty} \mathbf{C}^{(k)}(\mathbf{u}, l)
$$

where $\mathbf{C}^{(k)}$ is a term which contains expressions of precisely order $k$

$$
\mathbf{C}^{(k)} \propto \delta^{k}
$$

Similarly, we introduce the decompositions

$$
\mathrm{d} \mathbf{Q}=\sum_{k=2}^{\infty} \mathrm{d} \mathbf{Q}^{(k)}
$$

and

$$
\mathrm{d} \mathbf{P}=\sum_{k=2}^{\infty} \mathrm{d} \mathbf{P}^{(k)}
$$

We now proceed in two steps. We apply (30) with (31) and (25) on dP, but on both sides we take only the term of the order $\delta^{k}$.

Starting from (43), we define $g_{\nu}^{\left(k-k^{\prime}\right)}$ as a function which is precisely of order $\delta^{k-k^{\prime}}$ and $\mathrm{d} \overline{\mathrm{Q}}^{\left(k^{\prime}\right)}$ as a function being precisely of order $k^{\prime}$. We put

$$
\begin{aligned}
\Delta_{-}^{(u)(k)} \mathbf{f}= & \sum_{k^{\prime}=0}^{k} \sum_{\nu=0}^{l-l^{\prime}} g_{\nu}^{\left(k-k^{\prime}\right)}\left(\mathbf{u}_{l}, \ldots, \mathbf{u}_{l^{\prime}-1}\right) \mathrm{d} \overline{\mathbf{Q}}^{\left(k^{\prime}\right)} \\
& \times\left(\ldots, l^{\prime}+\nu\right)
\end{aligned}
$$

which is obviously of order $\delta^{k}$. After these preparations the final formula for $\mathbf{C}^{(k)}$ is given by 


$$
\begin{aligned}
\mathbf{C}^{(k)}= & \left\{\Delta_{-}^{(l)}\left(1+\Lambda_{s} \mathrm{~d} l\right)-\Lambda_{s} \mathrm{~d} l\right\}^{-1} \\
& \times \sum_{k_{1}+\cdots+k_{\nu}=k^{\prime}} \prod_{i=1}^{\nu}[\ldots]_{k_{i}} \mathrm{~d} \mathbf{P}^{\left(k-k^{\prime}\right)}
\end{aligned}
$$

together with

$$
\begin{aligned}
{[\ldots]_{k_{i}}=} & -\left(1+\Lambda_{s} \mathrm{~d} m\right) \Delta^{(u)\left(k_{i}\right)} T_{-}^{(l)} \\
& \times\left\{\Delta_{-}^{(l)}\left(1+\Lambda_{s} \mathrm{~d} l\right)-\Lambda_{2} \mathrm{~d} l\right\}^{-1} .
\end{aligned}
$$

We are now in a position to formulate the slaving principle for discrete (noisy) maps. According to this principle, we can express $\mathbf{s}$ by the sum (53), where the individual terms of order $\delta^{k}$ are explicitly given by (58) and (59). Note in particular that this result involves $\mathbf{u}_{l}$ only at a finite number of retarded time steps. For most practical applications it is sufficient to limit the sum over $k$ to the first leading terms. Because of the central role the slaving principle plays in the continuous time case we may expect a similar range of applications in the discrete time case. The next steps may consist in the discussion of normal forms or of typical cases of discrete maps of few variables.

\section{References}

[1] S. Grossmann and S. Thomae (1977). Z. Naturforschung 32A, 1353.

[2] H. Haken (1987). Advanced Synergetics, 2nd ed., Springer, Berlin.

[3] A.S. Mikhailov (1990). Foundations of Synergetics I, Springer, Berlin. 




Advances in

Operations Research

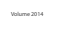

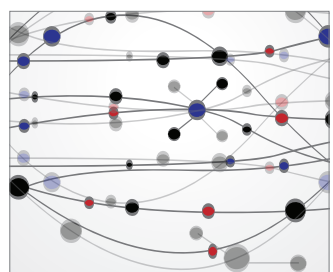

\section{The Scientific} World Journal


International Journal of

Mathematics and

Mathematical

Sciences
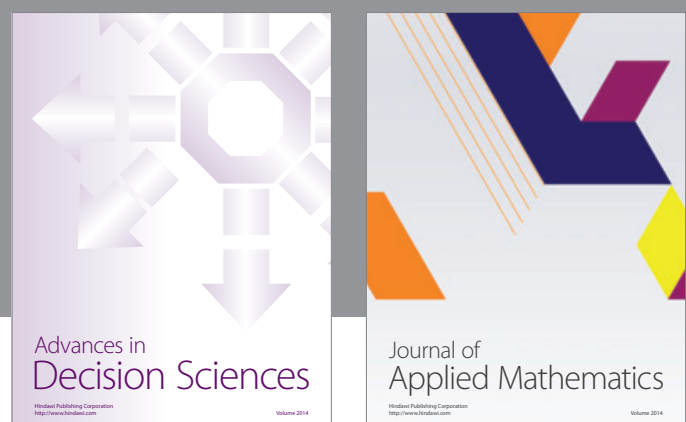

Journal of

Applied Mathematics


Submit your manuscripts at http://www.hindawi.com
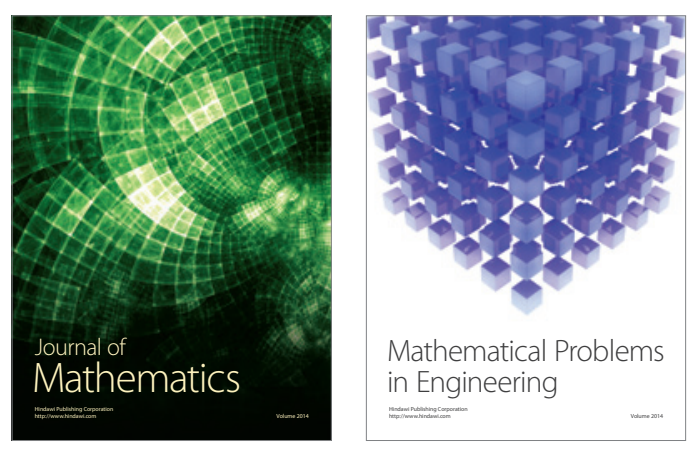

Mathematical Problems in Engineering
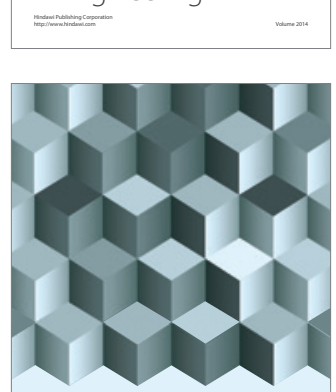

Journal of

Function Spaces
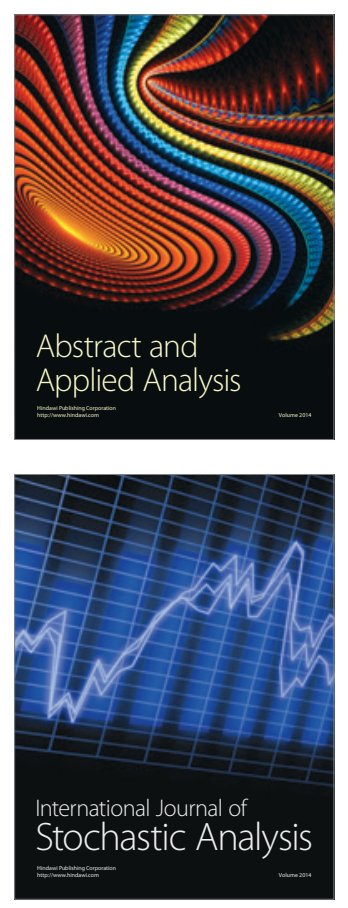



ournal of

Probability and Statistics

Promensencen
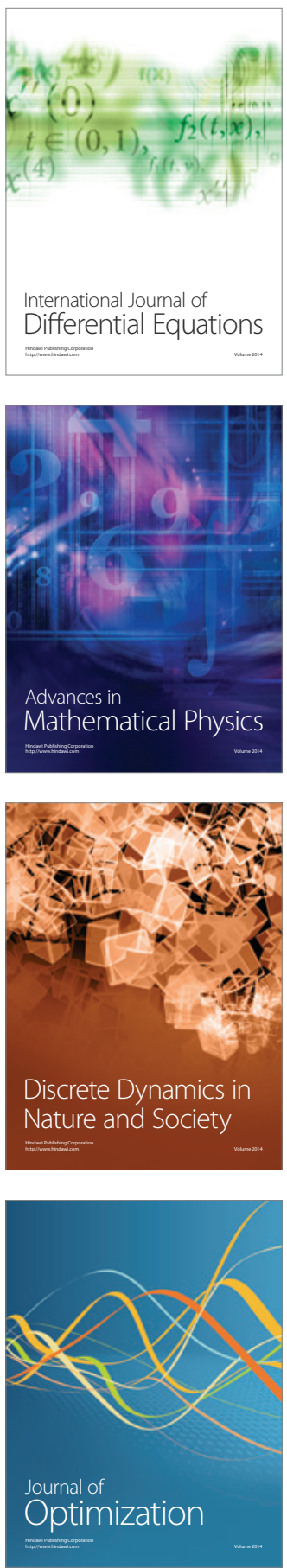